\title{
Huminstoffe in Sedimentporenwässern ausgewählter Bundeswasserstraßen
}

\author{
Elke Zwirnmann
}

Eingegangen: 11. März 2009/Akzeptiert: 29. April 2009/Online veröffentlicht: 21. Mai 2009

(C) Springer-Verlag 2009

\begin{abstract}
Zusammenfassung Huminstoffe (HS) sind ein wesentlicher Bestandteil des organischen Kohlenstoffs in Gewässern. Ihr Charakter und ihre Eigenschaften werden durch den Ursprung und die Art der Entstehung bestimmt. Die Huminstoffe sind nicht ausschließlich refraktäre Verbindungen, sondern sie sind an verschiedenen Stoffkreisläufen im Gewässer beteiligt. Da die Huminstoffe keiner direkten Analytik zugänglich sind, werden verschiedene Methoden angewendet, um sie zu charakterisieren. Das hier vorgestellte LC-OCD-Verfahren (Liquid Chromatography - Organic Carbon Detection) ist eine Ausschlusschromatografie mit einer online UV- und Kohlenstoffbestimmung. Damit können Kohlenstofffraktionen, z. B. Huminstoffe, im Wasser nach ihrer Molekülgröße unterschieden werden. In Sedimentporenwässern von Elbe, Rhein, Donau, Oder und der Müritz-Elde-Wasserstraße wurde der Huminstoffanteil bestimmt. Bis auf wenige Ausnahmen beträgt er zwischen 50 und $67 \%$ des gelösten organischen Kohlenstoffes. Die Fraktion der hochmolekularen Stoffe beträgt zwischen 10 und $34 \%$ und die der niedermolekularen Verbindungen zwischen 7 und $37 \%$. Die spezifische UV-Absorption (SUVA) der HS-Fraktion ist ein Maß für den Anteil der ungesättigten C-Bindungen und lässt im Zusammenhang mit dem Molekulargewicht auf den Humifizierungsgrad und das Alter der Huminsäuren schließen. Die Verteilung des gelösten organischen Kohlenstoffs auf die verschiedenen Fraktionen zeigt, dass in den Sedimentporenwässern Um- und Abbauprozes-
\end{abstract}

Verantwortliche Herausgeber: Jan Schwarzbauer - Peter Heininger · Evelyn Claus

E. Zwirnmann $(\bowtie)$

Leibniz Institut für Gewässerökologie und Binnenfischerei

Zentrales Chemielabor,

Müggelseedamm 301, 12587 Berlin, Deutschland

E-Mail: zwirnmann@igb-berlin.de se stattfinden, an denen sowohl hochmolekulare pflanzliche Bestandteile (Cellulose, Lignine) als auch biologische Abbauprodukte wie Aminosäuren oder Zucker beteiligt sind.

Schlüsselwörter Gelöster organischer Kohlenstoff · Huminstoffe · Größenausschlusschromatografie · Sedimentporenwasser

\section{Humic substances in sediment porewaters of selected waterways}

Abstract Humic substances are an important component of organic carbon in natural waters. Their character and properties are determined by the sources and the processes of their origin. Humic substances are not exclusively refractory compounds, but they are involved in several transformation processes in the water. It is not possible to directly analyze humic substances, therefore several methods were applied for their characterization. The presented LC-OCDtechnique is a size-exclusion-chromatography with online UV- and carbon detection. Carbon fractions, e.g. humic substances were differentiated by their molecular size. The portion of humic substances in sediment porewaters of the rivers Elbe, Rhine, Danube, Oder, Müritz-Elde-Waterway was determined. With only a few exceptions it ranges from 50 to $67 \%$ of the dissolved organic carbon. The high molecular weight fraction accounts for 10 to $34 \%$, and the fraction of the low molecular weight substances was from 7 to $37 \%$. The ratio between the spectral absorption coefficient $(254 \mathrm{~nm})$ and the organic carbon is called SUVA $(\mathrm{L} / \mathrm{mg} \times \mathrm{m})$ and is an inidicator of the proportion of unsaturated bonds in the humic substances.

Both high molecular herbal components (e.g. cellulose, lignin) and biological degradiation products (e.g. amino 
acids, sugar) are involved in sediment porewater transformation and degradiation processes. This demonstrates the distribution of the dissolved organic carbon between the fractions.

Keywords Dissolved organic carbon - Humic substances · Sediment porewater $\cdot$ Size-exclusion chromatography

\section{Problemstellung}

Der gelöste organische Kohlenstoff in Gewässern besteht aus einer Vielzahl organischer Verbindungen. Mehr als die Hälfte davon sind Huminstoffe, die wiederum ein sehr komplexes Gemisch darstellen. Der Charakter und die Eigenschaften der Huminstoffe werden durch ihren Ursprung und die Art ihrer Entstehung bestimmt. Die ursprüngliche Annahme, dass es sich dabei um refraktäre, d.h. mikrobiellem Abbau nicht zugängliche Verbindungen handelt, wurde in vielen Untersuchungen widerlegt (Steinberg 2003). Beispiele für ihre Beteiligung an den Stoffkreisläufen in Gewässern sind die Beeinflussung des Säurestatus, die Veränderung der Bioverfügbarkeit von Schwermetallen (Meinelt et al. 2001) und Xenobiotika (Perminova et al. 2001) oder Veränderungen über photolytische Reaktionen (Paul et al. 2004). Der komplexe Aufbau der Huminstoffe lässt keine direkte Analyse zu. Für ihre Charakterisierung werden verschiedene Methoden und Verfahren herangezogen, wie spektroskopische Methoden, Titration der Säuregruppen, Fraktionierungen z. B. nach Molekülgrößen oder Lösungseigenschaften.

\section{Ziel}

Mit dem LC-OCD-Verfahren (Liquid Chromatography Organic Carbon Detection) steht ein Verfahren zur Verfügung, mit dem ohne aufwändige Probenvorbereitung oder -anreicherung der Anteil der Huminstoffe in Wässern bestimmt werden kann. Außerdem können aufgrund ihrer Molekülgröße zwei weitere Kohlenstofffraktionen unterschieden werden: hochmolekulare organische Verbindungen sowie niedermolekulare organische Substanzen. In Sedimentporenwässern verschiedener Bundeswasserstraßen wurden die Kohlenstofffraktionen bestimmt und die Ergebnisse verglichen.

\section{Material und Methoden}

Probenahme: Die Proben wurden von der Bundesanstalt für Gewässerkunde zur Verfügung gestellt. Die Probenahme für die untersuchten Sedimente erfolgte bis auf eine Ausnahme mit einem van-Veen-Greifer aus den oberen $10 \mathrm{~cm}$ der Sedimente. Das Porenwasser wurde durch Zentrifugieren und anschließendem Filtrieren durch 0,45 $\mu \mathrm{m}$ Membranfilter (Celluloseacetat, Firma Sartorius, Göttingen) gewonnen. Alle Proben wurden bei $4{ }^{\circ} \mathrm{C}$ transportiert und gelagert.

Methoden: In dem von Huber u. Frimmel (1996) entwickelten LC-OCD-Verfahren (Liquid Chromatographic Organic Carbon Detection) wird der DOC im Wasser über die Molekülgröße aufgetrennt. Als Eluent wird $29 \mathrm{mM}$ Phosphatpuffer mit einer Fließgeschwindigkeit von $1 \mathrm{~mL} / \mathrm{min}$ verwendet. Die verschiedenen Fraktionen werden in einem Dünnfilmreaktor mit UV-Licht $(185 \mathrm{~nm})$ zu Kohlendioxid oxidiert und anschließend in einer Infralyteinheit detektiert. Mithilfe der Auswertesoftware FIFFIKUS werden drei Fraktionen des chromatografierbaren organischen Kohlenstoffes (CDOC) zusammengefasst: hochmolekulare Substanzen (HMWS - z. B. Polysaccharide), Huminstoffe (HS) und niedermolekulare Substanzen (LMWS - z.B. organische Säuren, Aminosäuren, Zucker). Für die Huminstofffraktion werden zwei weitere charakteristische Größen berechnet: die spezifische UV-Absorption $(254 \mathrm{~nm})$ bezogen auf $\mathrm{mg} / \mathrm{L} \mathrm{C}(\mathrm{SUVA}$ in $\mathrm{L} / \mathrm{mg} \times \mathrm{m}$ ), die ein Maß für den Anteil der ungesättigten Verbindungen in diesem Huminstoff ist, sowie das Molekulargewicht im Peakmaximum $\left(\mathrm{M}_{\mathrm{p}}\right.$ in $\mathrm{g} / \mathrm{mol}$ ). Eine zusätzliche Information gibt ein Peak im UV-Chromatogramm im Bereich der Retentionszeiten der HWMS. Dabei handelt es sich um anorganische Kolloide, z.B. Eisen- und Aluminiumkolloide.

\section{Ergebnisse und Diskussion}

In Abb. 1 ist beispielhaft ein Chromatogramm des Sedimentporenwassers der Elbe bei Lauenburg dargestellt. Das obere Chromatogramm ist die Linie des Kohlenstoffdetektors, das untere die des UV-Detektors. Der erste Peak zeigt die Fraktion der höhermolekularen Substanzen, die oft auch unter dem Begriff Polysaccharide zusammengefasst werden. Der Peak in der UV-Linie bei dieser Retentionszeit ist auf die oben genannten anorganischen Kolloide zurückzuführen. Der mittlere Peak stellt die Huminstoffe dar, und der nachfolgende Peak zeigt niedermolekulare organische Säuren an, die auch im UV-Chromatogramm zu sehen sind, da sie ungesättigte Verbindungen enthalten. Alle nachfolgenden Peaks sind neutrale niedermolekulare Verbindungen. Bei den Berechnungen werden die beiden letzten Fraktionen unter dem Begriff niedermolekulare Verbindungen (LMWS) zusammengefasst. In Tabelle 1 sind die Ergebnisse als Mittelwerte aus drei Wiederholungsmessungen für die verschiedenen stichprobenartig ausgewählten Probenahmestellen zusammengefasst. Die Anzahl der Probenahmestellen war nicht einheitlich für die einzelnen Bundeswasserstraßen. In der Elbe nehmen die Kohlenstoffgehalte im Sedimentporenwasser flussabwärts zu, und gleichzeitig steigt auch der Anteil der höhermolekularen Fraktion. Eine Ursache dafür 
Abb. 1 LC-OCD-Chromatogramm der Elbe bei $\mathrm{km}$ 570 (MWS: Hochmolekulare Substanzen, $H S$ : Huminstoffe, $L M W A$ : Niedermolekulare Säuren, LWS: Niedermolekulare neutrale Substanzen)

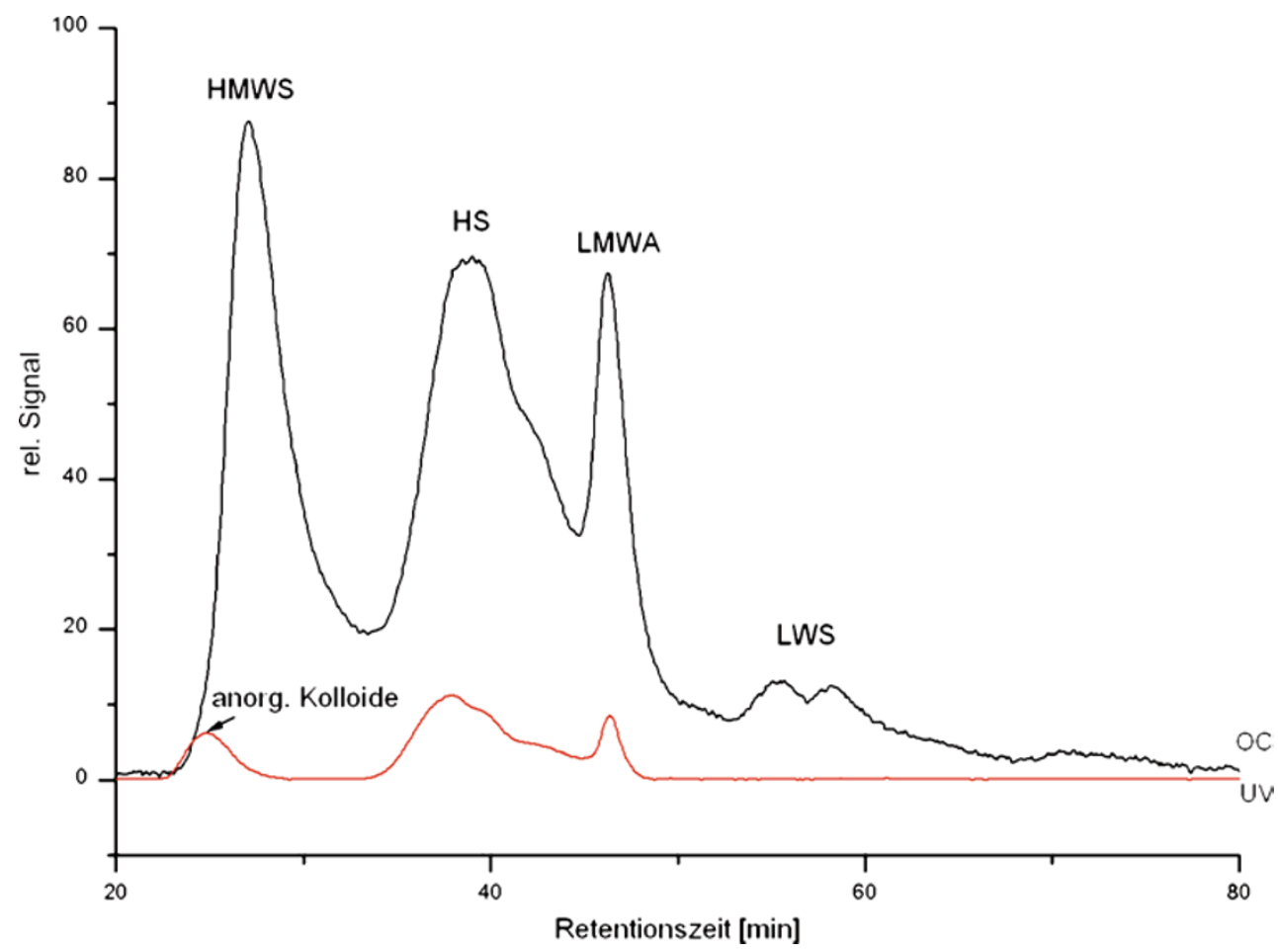

Tabelle 1 Kohlenstofffraktionen in Sedimentporenwässern

\begin{tabular}{|c|c|c|c|c|c|c|c|c|c|}
\hline Wasserstraße & Fluss-km & Ort & $\begin{array}{c}\text { CDOC } \\
(\mathrm{mg} / \mathrm{L} \mathrm{C})\end{array}$ & $\begin{array}{c}\text { HMWS } \\
\%\end{array}$ & $\begin{array}{l}\text { HS } \\
\%\end{array}$ & $\begin{array}{c}\text { LMWS } \\
\%\end{array}$ & $\begin{array}{c}\text { SUVA } \\
(\mathbf{L} / \mathbf{m g} \times \mathbf{m})\end{array}$ & $\begin{array}{c}M_{p} \\
(g / m o l)\end{array}$ & $\begin{array}{c}\text { Anorg. } \\
\text { Kolloide } \\
(1 / \mathbf{m})\end{array}$ \\
\hline \multirow[t]{2}{*}{ Rhein } & 591 & Ehrenbreitstein & 8,3 & 17 & 52 & 31 & 2,7 & 3606 & 1,6 \\
\hline & 864 & Bimmen & 12,9 & 14 & 64 & 22 & 2,8 & 2926 & 0,8 \\
\hline \multirow[t]{4}{*}{ Elbe } & 252 & Alte Elbe & 15,1 & 23 & 60 & 17 & 4,2 & 6321 & 6,5 \\
\hline & 314 & Schönebeck & 18,5 & 30 & 54 & 17 & 3,9 & 3920 & 25,0 \\
\hline & 319 & Magdeburg & 35,4 & 54 & 35 & 12 & 3,8 & 5428 & 30,4 \\
\hline & 570 & Lauenburg & 39,1 & 45 & 44 & 11 & 4,1 & 3444 & 176 \\
\hline \multirow[t]{2}{*}{ Donau } & 2203 & Jochenstein & 24,3 & 11 & 43 & 46 & 2,9 & 3772 & 1,9 \\
\hline & 2403 & Bad Abbach & 10,8 & 27 & 57 & 16 & 2,9 & 4622 & 2,6 \\
\hline \multirow{2}{*}{$\begin{array}{l}\text { Müritz-Elde- } \\
\text { Wasserstraße }\end{array}$} & 1 & Dömitz & 1,6 & 24 & 59 & 17 & 3,0 & 3696 & 2,0 \\
\hline & 73 & Parchim & 12,3 & 15 & 68 & 17 & 3,7 & 4097 & 3,2 \\
\hline Oder & 655 & Hohenwutzen & 11,4 & 25 & 55 & 20 & 3,5 & 3852 & 4,9 \\
\hline
\end{tabular}

CDOC: Chromatografierbarer gelöster organischer Kohlenstoff

HMWS: Hochmolekulare Substanzen

HS: Huminstoffe

LMWS: Niedermolekulare Substanzen

SUVA: Spezifischer UV-Absorptionskoeffizient

$\mathrm{M}_{\mathrm{p}}$ : Molekulargewicht im Peakmaximum des HS-Peaks

könnte eine erhöhte Algenproduktion im Flussverlauf sein. Dagegen nimmt der Huminstoffanteil flussabwärts ab und liegt zwischen 60 und $35 \%$ des DOC. Auffallend ist noch der außergewöhnlich hohe Anteil der anorganischen Kolloide bei Lauenburg, km 570 (Abb. 1). Die Zusammensetzung des Sedimentes und des Porenwassers wird von vielen Faktoren bestimmt. Dazu gehören z.B. die Morphologie, die Wasserführung, die Einleitung von Abwässern sowie Stoffeinträge aus dem Einzugsgebiet (Sachse 2004). Die registrierten Unterschiede der Kohlenstofffraktionen entlang eines Flussverlaufs lassen sich daher nur schwer interpretieren, weil Abbau- und Umbauprozesse von Einträgen und selektivem Transport überlagert werden. Deshalb wurde bei den Flüssen mit nur zwei Stichproben keine weitere Bewer- 
tung vorgenommen. Für detailliertere Bewertungen ist eine wesentliche höhere Anzahl Proben sowie die Bestimmung weiterer Parameter nötig.

\section{Ausblick}

Mit dem LC-OCD-Verfahren ist es möglich, den gelösten organischen Kohlenstoff in Wasser nach der Molekülgröße aufzutrennen. Seine Verteilung auf die verschiedenen Fraktionen zeigt, dass in den Sedimentporenwässern Um- und Abbauprozesse stattfinden, an denen sowohl hochmolekulare pflanzliche Bestandteile (Cellulose, Lignine) als auch biologische Abbauprodukte wie Aminosäuren oder $\mathrm{Zu}$ cker beteiligt sind. Aussagen über Wirkmechanismen der Huminstoffe können nur im Zusammenhang mit weiteren Parametern wie pH-Wert, Redoxpotenzial, Metallkonzentrationen, Metallbindungsvermögen der HS und zusätzlichen Laborexperimenten gemacht werden.

\section{Literatur}

Huber SA, Frimmel FH (1996) Gelchromatographie mit Kohlenstoffdetektion (LC-OCD): Ein rasches und aussagekräftiges Verfahren zur Charakterisierung hydrophiler organischer Wasserinhaltsstoffe. Wasser 86:277-290

Meinelt T, Playle RC, Pietrock M, Burnison BK, Wienke A, Steinberg CEW (2001) Interaction of cadmium toxicity in embryos and larvae of zebrafish (Danio rerio) with calcium and humic substances. Aquat Toxicol 54:205-215

Paul A, Hackbarth S, Vogt RD, Röder B, Burnison BK, Steinberg CEW (2004) Photogeneration of singlet oxygen by humic substances: Comparison of humic substances of aquatic and terrestrial origin. Photochem Photobiol Sci 3:273-280

Perminova IV, Grechishcheva NY, Kovalevskii DV, Kudryavtsev AV, Petrosyan VS, Matorin DN (2001) Quantification and prediction of the detoxifying properties of humic substances related to their chemical binding to polycyclic aromatic hydrocarbons. Environm Sci Technol 35:3841-3848

Sachse A (2004) Herkunft, Menge und Struktur des gelösten organischen Kohlenstoffs (DOC) in Oberflächengewässern. Dissertation, Humboldt-Universität zu Berlin

Steinberg CEW (2003) Ecology of Humic Substances in Freshwaters. Springer, Berlin Heidelberg 